\title{
Micro- and macroscopic characterizations of the viscoelastic fracture of resin-based fibre composites
}

\author{
S.Y. Zhang \\ Institute of Mechanics, Chinese Academy of Sciences, 15 Zhong Guan Cun Road, Beijing 100080, China
}

Received 19 January 1998; received in revised form 17 March 1998; accepted 19 March 1998

\begin{abstract}
Micro- and macroscopic characterizations of the viscoelastic fracture of a unidirectional carbon-fibre-reinforced epoxy composite are presented. First, the micro-cracking behavior of the material is studied by the use of scanning electron microscopy; the in situ creep cracking process is observed and the crack propagation is measured. In order to obtain insight into the mechanisms of the observed creep cracking, macroscopic investigations were also carried out. Finite-element method simulations were carried out to calculate the stress distribution and the variation of stresses with time. A theoretical analysis of the orthotropy of viscoelastic fracture behavior of the material is also conducted. (C) 1999 Elsevier Science Ltd. All rights reserved.
\end{abstract}

Keywords: A. Polymer-matrix composite; C. Finite element analysis; D. Scanning electron microscopy; Creep fracture; Time-dependent properties

\section{Introduction}

As a consequence of the viscoelasticity of the polymeric matrix, the behavior of matrix-dominated fracture, i.e., cracking through the matrix, is obviously timedependent; delayed crack initiation and crack extension with time are the two typical viscoelastic fracture phenomena. Investigation of this problem is meaningful for design and application of structures composed of this kind of material and has been carried out for longer than twenty years by many authors. For instance, the delayed fracture problem was dealt with by Knauss [1] and the theory of crack initiation and propagation in a viscoelastic medium was proposed by Schapery [2] in the 1970s; both of the two pieces of research are based on linear viscoelastic constitutive relationships. In the early 1980s, research on the fracture of non-linear viscoelastic media was carried out. Some results from a generalized J-integral approach were reviewed and the theory of crack growth in power-law materials was given by Schapery [3]. Recently, slow crack propagation in power-law non-linear viscoelastic materials was predicted by a perturbation method by Zhang et al. [4]. Moreover, although many papers on theoretical studies in this area can be found in the literature, few experimental studies have been done so far and the established theoretical models are yet to be verified experimentally. In addition to the time-dependency of fracture, another feature of the viscoelastic fracture of fibre-reinforced polymer composites is the anisotropy of their fracture behaviour. The theory of orthotropically viscoelastic deformation and dynamic stability of the material has been investigated by Chandiramani et al. [5]. However, no research work on anisotropy of viscoelastic fracture behaviour has been seen and the time-dependency of the anisotropy of fracture behavior is yet to be investigated.

The paper consists of two parts. In the first part, an experimental study on the creep fracture of a unidirectional carbon-fiber-reinforced epoxy composite with notches was carried out by scanning electron microscopy (SEM); and the crack initiation and variation of crack length, $a$, with time were observed. It was found that under constant load, the crack initiates from the root of a notch and grows parallel to the fibers with time continuously. To understand the time-dependency of fracture of the composite material is of significance for the designers of composite structures.

The second part of the paper is devoted to the macrocharacterization of the delayed cracking observed with the SEM and attempts are made to explain the mechanisms of the viscoelastic cracking in this case study. First, a finite-element simulation was performed by using viscoelastic constitutive relationship, revealing the variation pattern of the stress concentrations at notch roots with time. The time-dependency of the orthotropy of fracture behaviour of the material was then analyzed. 
Numerical evaluation of the preference of the cracking direction was finally attempted. The variation with time of the ratio between the fracture toughness in the longitudinal direction to that in the transverse direction was predicted and the curve of the toughness ratio versus time is given; that may be helpful for understanding the time-dependent fracture phenomenon observed.

\section{Experimental}

\subsection{Material and specimen}

The material tested is a unidirectional composite consisting of T300 carbon fibre in epoxy resin. This composite sheet was manufactured by laying-up 7 prepreg layers. The thickness of one layer is about 0.125 $\mathrm{mm}$. This composite material is one of the typical materials used in the astronautical and aeronautical fields.

The configuration of the three-point bend beam specimen is shown schematically in Fig. 1. Two slender notches were cut normal to the fibers and symmetrically to the center line of the specimen.

\subsection{Test procedure}

The main test procedure was as follows:

- Mount the specimen on the testing rig in the loading stage of SEM;

- Load the specimen step by step quickly, so as to minimize the effect of viscoelasticity;

- When the load reaches the predetermined value, stop raising the load and keep it constant. The load value was determined according to the averaged failure strength and is equal to $9.8 \mathrm{~N}$, which is $85 \%$ of the value of the failure load.

- Watch the end of the notch, when crack initiates, take a photograph. The crack will then propagate further and further as the time goes on; a series of photographs are taken continually to record the crack length. Once the crack grows, the load will drop a little: then modify the load as soon as possible, so as to keep it approximately constant.

- It is observed that under constant load, two cracks first initiate from the inner corner of the two notches and propagate along the fibres towards the center

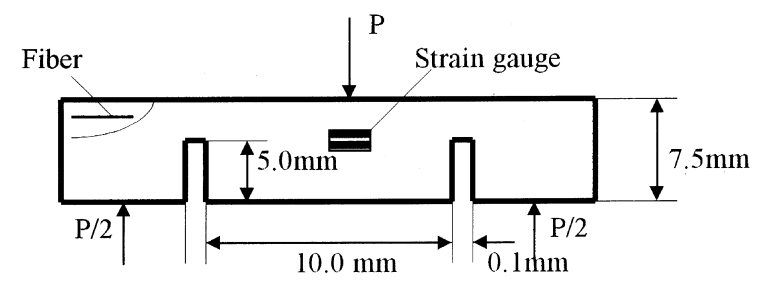

Fig. 1. Schematic drawing of the specimen and loading configuration. line of the beam, so that the two crack tips are getting nearer and nearer, and finally the specimen fails.

The experimental environment was un-controlled: room temperature in summer was about $27^{\circ} \mathrm{C}$ and the humidity was about $70 \%$.

\subsection{Experimental results}

Total 5 specimens were tested. For the sake of conciseness, only typical results will be given in the following.

\subsubsection{Photographs}

Fig. 2a illustrates the initiation of a crack from the root of a notch, Fig. $2 b$ shows the propagating crack, and the photograph in Fig. 2c is an image of a crack at high magnification. It can be seen from Fig. 2c that for most of the crack length, the fiber surface is covered with adhering matrix debris; while for remainder, the fiber surface is clean and smooth, so that it may be judged that matrix cracking and interfacial debonding are the main fracture mechanisms of creep fracture. However, there are occasions that when crack splits the fiber in the longitudinal direction.

\subsubsection{Curve of crack length versus time}

Fig. 3 is a typical plot of crack length versus time recorded for specimen No.3. It can be seen that the fracture behavior is time dependent and the crack propagates slightly more rapidly in the earlier period of time than at later times. This tendency resembles that of a creep-strain curve under constant stress.

\section{FEM simulations}

\subsection{Viscoelastic constitutive relationship}

The viscoelastic constitutive relationship of the composite material was derived from those of the matrix and the fibres. The time-dependent Young's modulus of the matrix is given in Ref. [5], and can be simulated by using the Standard Linear Solid model (i.e., 3-parameter model):

$$
E(t)=0.552+1.18 \times \mathrm{e}-0.4115 \times 10^{-3} t \mathrm{GPa}
$$

where, $t$ is in hours and $0 \leqslant t \leqslant 2000 \mathrm{~h}$. The constitutive relationship in Eq. (1) is measured at a temperature of $T=25^{\circ} \mathrm{C}$.

The fibres are assumed to be purely elastic and the material constants of $\mathrm{T} 300$ are:

$$
\begin{aligned}
& E_{L}=221.0 \mathrm{GPa}, \quad E_{T}=13.8 \mathrm{GPa}, \\
& G_{\mathrm{TL}}=9.0 \mathrm{GPa}, \quad v_{\mathrm{LT}}=0.27 .
\end{aligned}
$$



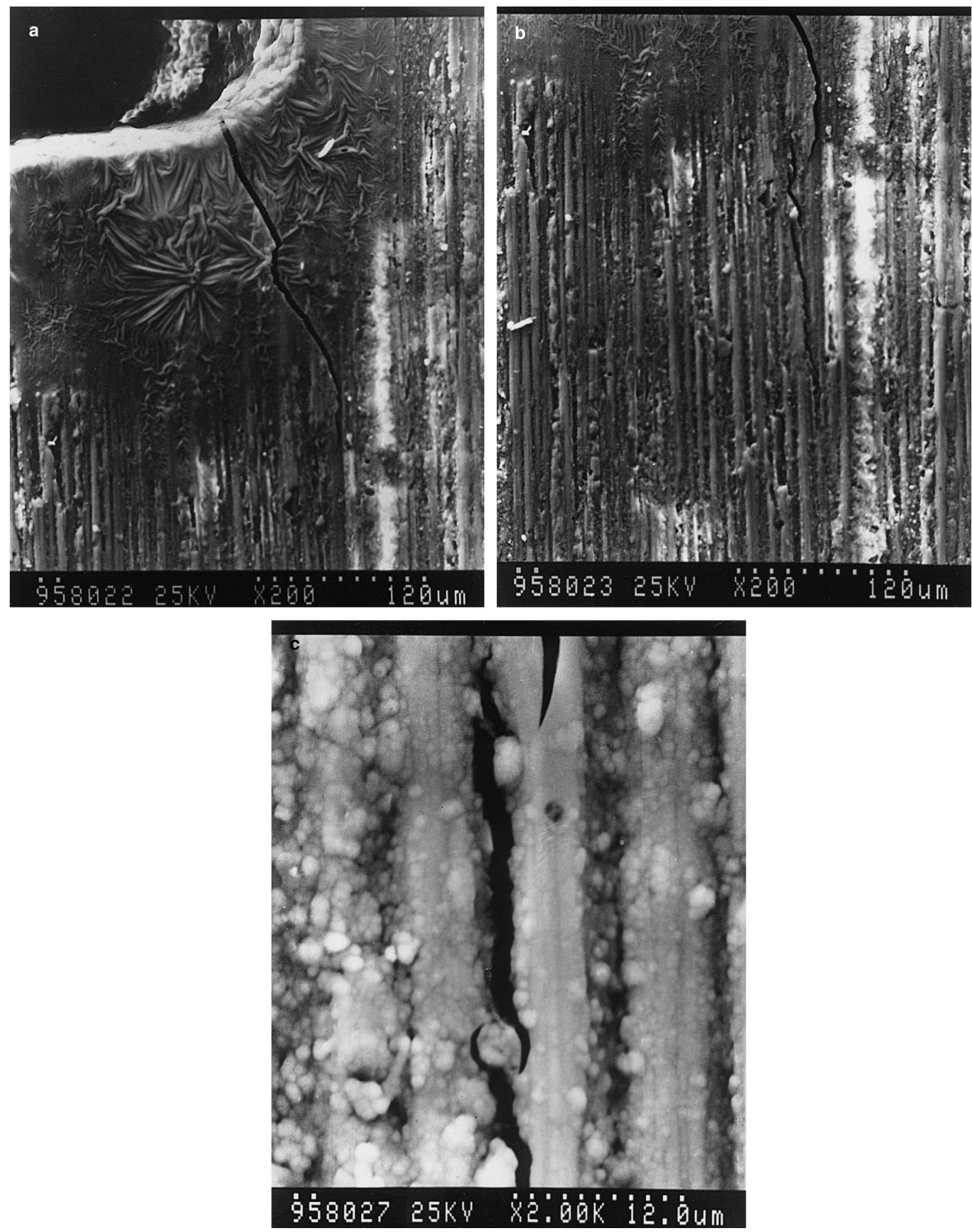

Fig. 2. (a) Photograph showing crack initiation from the end of notch; (b) Photograph showing crack in propagation; (c) Photograph with large magnification showing details of debonded interface.

The well-known rule of mixtures for composites is used to derive the orthotropically viscoelastic properties of the composite. In so doing, it is assumed that the Poisson's ratios of the fiber and matrix are constants; they are $v_{m}=0.38, v_{f}=0.27$. The fibre volume fraction is 0.6 . For the sake of conciseness, the expressions and curves for $E_{L}(t)$ and $E_{T}(t)$ are omitted.

\subsection{Elastic analysis}

The elastic analysis was performed by using the FE method to calculate the stress distribution in the specimen. Emphasis is laid on the stress concentration at the notch root. The material constants were derived from the viscoelastic constitutive relationship of the compo- 


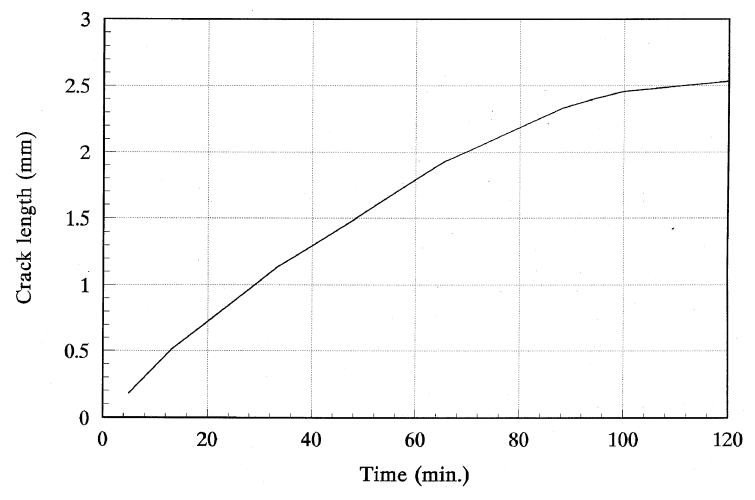

Fig. 3. Plot of crack length versus time for specimen \#3.

site obtained in the previous section and are:

$E_{11}=133.3 \mathrm{GPa}, \quad E_{22}=3.75 \mathrm{GPa}$,

$G_{12}=1.50 \mathrm{GPa}, \quad v_{12}=0.32$.

Actually, these data are pertinent to the initial elastic properties of the composite, i.e. by setting $t=0$ in Eq. (1) and by using the data of Eqs. (1) and (2) and the rule of mixtures, it can be proved that the data in Eq. (3) are just the initial values of the viscoelastic properties.

Eight-node quadrilateral isoparametric elements were used. The FE mesh pattern is as shown in Fig. 4. Half of the specimen was calculated by virtue of symmetry. The stress distributions along the longitudinal line through the ends of the notches were calculated, and the stress curves are given in Fig. $5 \mathrm{a}-\mathrm{c}$ for $\sigma_{x x}, \sigma_{y y}, \sigma_{x y}$ respectively. It should be pointed out that the stress values are the element centre stresses. It can be seen that there are considerable stress concentrations at the root of the notch, which may cause creep cracking.

\subsection{Viscoelastic analysis}

The orthotropically viscoelastic material properties obtained in Section 3.1 are mainly for the use in viscoelastic analysis. The mesh shown in Fig. 4 was also used here. The variations of $\sigma_{x x}, \sigma_{y y}, \sigma_{x y}$ with time were evaluated. The stresses are calculated for the element at the left hand corner of the notch root, which exhibits

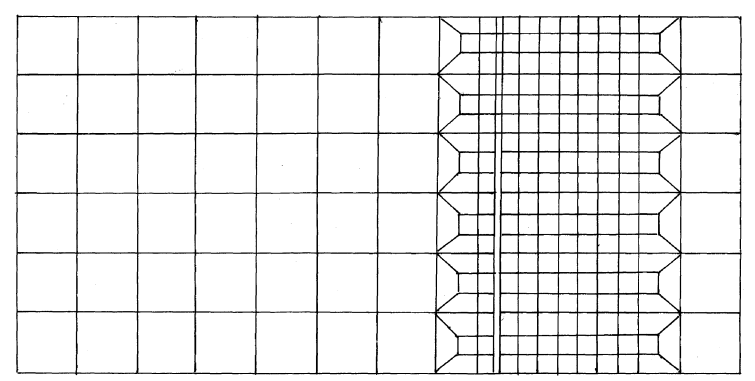

Fig. 4. FEM mesh pattern. the maximum stress concentration. The results are shown as the curves in Fig. 6a-c. It can be indicated that under the constant applied load, the absolute values of the three stress components are increasing with time, which may, qualitatively, be the explanation of the creep cracking observed in the SEM experiments.

\section{Orthotropy of viscoelastic fracture toughness}

As mentioned earlier, resin-based fiber composites behave both viscoelastically and anisotropically. The fracture behaviour of the material is viscoelastically orthotropic or orthotropically viscoelastic. Fracture orthotropy in unidirectional fibre composites is particularly remarkable. Cracks tend to grow along fibers rather than normal to them. The problems of crack propagation in unidirectional composites have been investigated by many researchers. Zhang et al [6], Ashby et al [7] and Maiti [8] have analyzed the Mode-I in-plane fracture toughnesses of the two cases of a crack parallel to the fibers and a crack normal to the fibers. They gave the relationship between the two fracture toughness.

The mechanism of creep cracking along the fibers may also be relevant to the orthotropy of the viscoelastic fracture toughness. For Mode-I fracture, the formulae of a singular stress field at the crack tip in the orthotropic materials can be expressed as [9]:

$$
\begin{aligned}
\sigma_{x x} & =\frac{K_{I}}{\sqrt{2 \pi r}} \operatorname{Re}\left\{\frac { \mu _ { 2 } \mu _ { 1 } } { ( \mu _ { 1 } - \mu _ { 2 } ) } \left[\frac{\mu_{2}}{\left(\cos \theta+\mu_{2} \sin \theta\right)^{1 / 2}}\right.\right. \\
& \left.\left.-\frac{\mu_{1}}{\left(\cos \theta+\mu_{1} \sin \theta\right)^{1 / 2}}\right]\right\}, \\
\sigma_{y y} & =\frac{K_{I}}{\sqrt{2 \pi r}} \operatorname{Re}\left\{\frac { 1 } { ( \mu _ { 1 } - \mu _ { 2 } ) } \left[\frac{\mu_{1}}{\left(\cos \theta+\mu_{2} \sin \theta\right)^{1 / 2}}\right.\right. \\
& \left.\left.-\frac{\mu_{2}}{\left(\cos \theta+\mu_{1} \sin \theta\right)^{1 / 2}}\right]\right\}, \\
\sigma_{x y} & =\frac{K_{I}}{\sqrt{2 \pi r}} \operatorname{Re}\left\{\frac { \mu _ { 2 } \mu _ { 1 } } { ( \mu _ { 1 } - \mu _ { 2 } ) } \left[\frac{1}{\left(\cos \theta+\mu_{1} \sin \theta\right)^{1 / 2}}\right.\right. \\
& \left.\left.-\frac{1}{\left(\cos \theta+\mu_{2} \sin \theta\right)^{1 / 2}}\right]\right\},
\end{aligned}
$$

where Re is the real part of the complex number; $\mu_{1}$ and $\mu_{2}$ (or their conjugated complex numbers $\mu_{1}$ and $\mu_{2}$ ) are two different complex roots of Eq. (7),

$a_{11} \mu^{4}-2 a_{16}^{3}+\left(2 a_{12}+a_{66}\right) \mu^{2}-2 a_{26} \mu+a_{22}=0$,

where $a_{i j}$ are the elements of the compliance matrix of the material. For orthotropic composites and when the crack is embedded in one of the symmetric planes, $a_{16}$ and $a_{26}$ are equal to zero.

For the case of cracking parallel to fibers, 

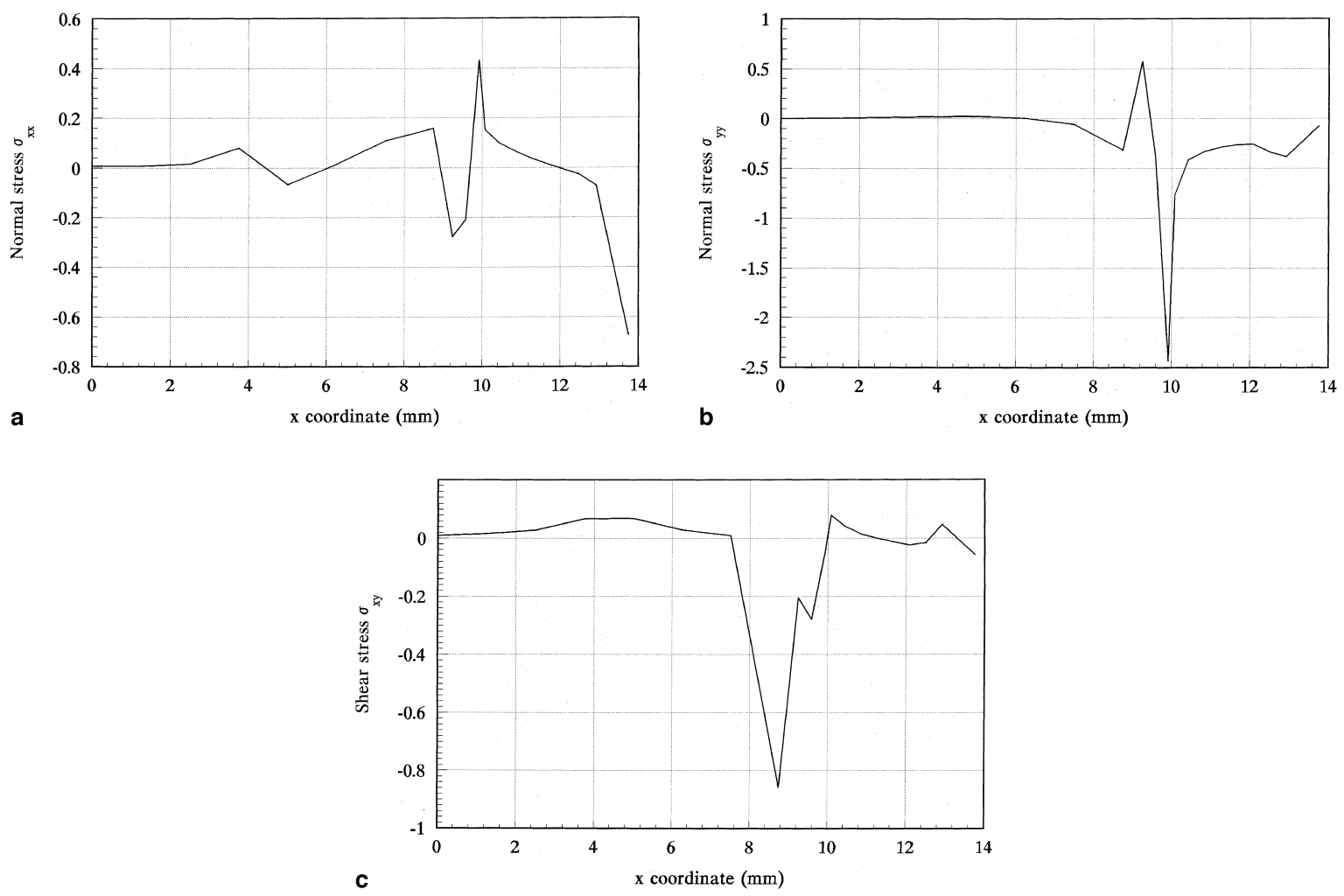

Fig. 5. (a) Distribution of $\sigma_{x x}$; (b) Distribution of $\sigma_{y y}$; (c) Distribution of $\sigma_{x y}$.
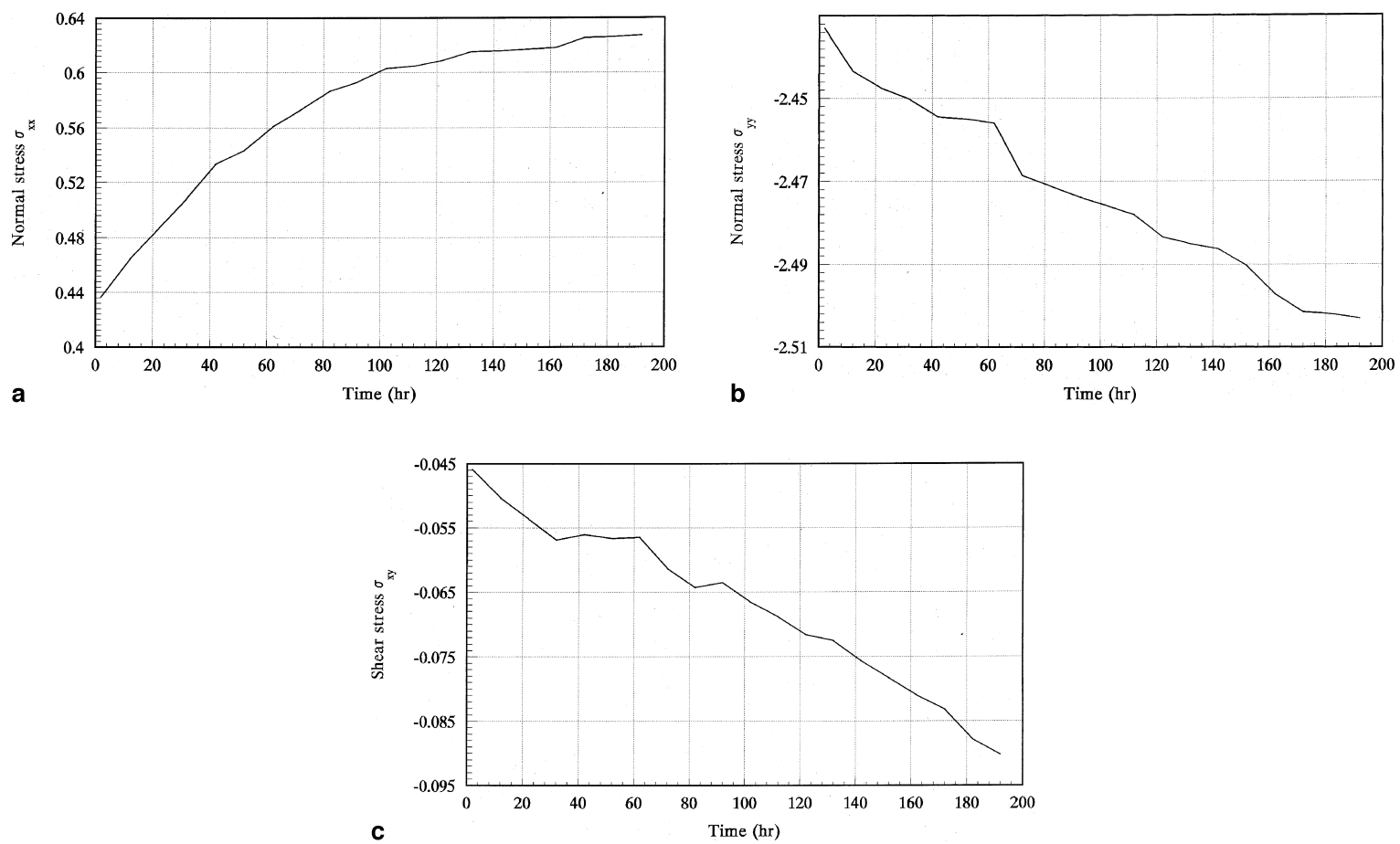

Fig. 6. (a) Variation of normal stress $\sigma_{x x}$ with time; (b) Variation of normal stress $\sigma_{y y}$ with time; (c) Variation of normal stress $\sigma_{x y}$ with time. 
$a_{11}=\frac{1}{E_{1}}, \quad a_{22}=\frac{1}{E_{2}}$,

$a_{66}=\frac{1}{G_{12}}, \quad a_{12}=\frac{-v_{12}}{E_{1}}=\frac{-v_{21}}{E_{2}}$.

Whereas, for cracking normal to the fibers,

$a_{11}=\frac{1}{E_{2}}, \quad a_{22}=\frac{1}{E_{1}}, \quad a_{66}=\frac{1}{G_{12}}$,

$a_{12}=\frac{-v_{12}}{E_{2}}=\frac{-v_{21}}{E_{1}}$.

Substituting Eqs. (8), (9) and (7) into Eqs. (4)-(6), the stress fields for the two crack configurations are obtained. Fracture can then be predicted by using a stress-based fracture criterion.

For cracking parallel to the fibers, it is postulated that $\sigma_{y y}$ is the only stress component which causes fracture, whereas for cracking normal to the fibers, the fracture is mixed-mode, the fracture is attributed to both $\sigma_{x x}$ and $\sigma_{x y}$. According to the point-stress fracture criterion [10], for the aligned crack, the onset of fracture occurs if the stress value satisfies the following condition:

$\sigma_{y y}^{a}=T=\frac{K_{\mathrm{IC}}^{a}}{\sqrt{2 \pi r_{0}}}$,

where $r_{0}$ is the characteristic dimension of the fracture process zone, $T$ is the transverse strength of the material and $K_{\mathrm{IC}}^{a}$ is the fracture toughness for the aligned crack.

For the perpendicular crack, the fracture condition is:

$\left[\frac{\sigma_{x x}^{n}}{T}\right]^{2}+\left[\frac{\sigma_{x y}^{n}}{S}\right]^{2}=1$

This is the modified Tsai-Hill criterion, in which the term in $\sigma_{y y}$ is omitted. In Eq. (11), $\sigma_{x x}^{n}$ and $\sigma_{x y}^{n}$ are the stress values at a point $r_{0}$ away from the crack tip; $S$ is the in-plane shear strength.

Combining Eqs. (10) and (11), and substituting Eqs. (4)-(6) into them, we obtained:

$$
\begin{aligned}
\frac{K_{\mathrm{IC}}^{n}}{K_{\mathrm{IC}}^{a}} & =\left\{\left[\frac{(A B)^{\frac{1}{2}}}{\sqrt{2}\left(A^{\frac{1}{4}}+B^{\frac{1}{4}}\right)}\right]^{2}\right. \\
& \left.+\left[\left(\frac{T}{S}\right) \frac{(A B)^{\frac{1}{4}}}{\sqrt{2}\left(A^{\frac{1}{4}}+B^{\frac{1}{4}}\right)}\right]^{2}\right\}^{-\frac{1}{2}},
\end{aligned}
$$

where $\left\{\begin{array}{l}A \\ B\end{array}\right\}=\frac{1}{2}\left[\frac{E_{2}}{G_{12}}-2 v_{21}\right]$

$$
\pm \frac{1}{2}\left[\left(\frac{E_{2}}{G_{12}}-2 v_{21}\right)^{2}-4 \frac{E_{2}}{E_{1}}\right]^{\frac{1}{2}} .
$$

When $\frac{T}{S} \rightarrow 0$ i.e. the shear strength $S$ is much larger than the transverse tensile strength $T$, Eq. (12) is reduced to:

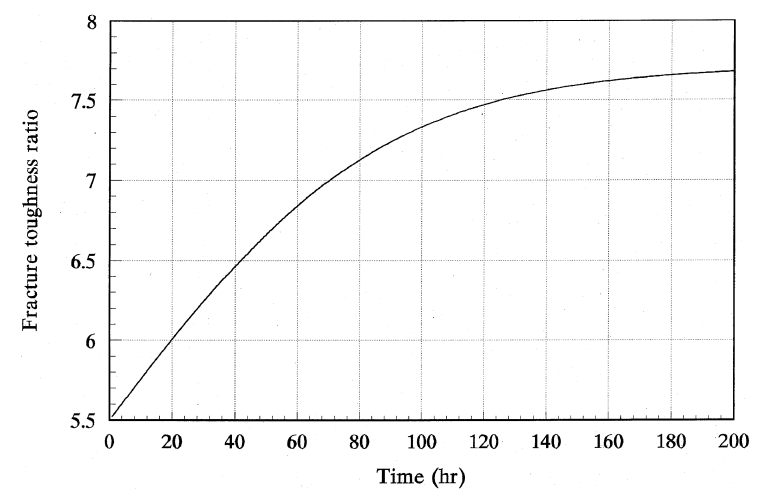

Fig. 7. Variation of fracture toughness ratio with time for $T / S=40.1 /$

$\frac{K_{\mathrm{IC}}^{n}}{K_{\mathrm{IC}}^{a}}=\sqrt{\frac{2}{A B}}\left(A^{\frac{1}{4}}+B^{\frac{1}{4}}\right)$

Eq. (13) can be found in Ref. [6,7], where the ratio of fracture toughnesses is constant, whereas in this paper it is time-dependent.

Numerical calculations were conducted for the plasticized epoxy carbon composite. The viscoelastic constitutive relationship given in Section 3.1 was also used here. It is assumed that $T=40.1 \mathrm{MPa}, S=67.2 \mathrm{MPa}$. Since the material properties of (8) and (9) are timedependent, the toughness ratio $K_{\mathrm{IC}}^{n} / K_{\mathrm{IC}}^{a}$ is also timedependent. The calculated result of the toughness ratio $K_{\mathrm{IC}}^{n} / K_{\mathrm{IC}}^{a}$ versus time is shown in Fig. 7. It is proved that the toughness ratio, $K_{\mathrm{IC}}^{n} / K_{\mathrm{IC}}^{a}$, increases with time; this implies that as time passes the composites will have a stronger tendency to fracture along the fibers rather than normal to them. This recognition is meaningful to the designer of the engineering structures composed of viscoelastic matrix composites.

\section{Concluding remarks}

The viscoelastic fracture of a unidirectional carbonfibre-reinforced epoxy composite has been studied both micro-experimentally and macro-analytically. The in situ observation with SEM revealed that the cracking behaviour is time-dependent. This phenomenon is difficult to predict exactly, in particular, for the phase of crack growth with time. The explanations given in the paper are semi-quantitative. A further investigation needs to be developed.

\section{Acknowledgements}

This research has been supported by the Chinese Sciences Foundation, and by LNM Chinese Academy of Sciences. The author is indebted to Dr. R.K.Y. Li of City University of Hong Kong. 


\section{References}

[1] Knauss WG. Delayed fracture-the Griffith problem for linearly viscoelastic materials. Int Jour Fract 1970;6(1):7-20

[2] Schapery RA. A theory of crack initiation and growth in viscoelastic medium: I. Theoretical development. Inter Jour Fract 1975;11(1):141-59

[3] Schapery RA. Nonlinear fracture analysis of viscoelastic composite materials based on a generalized $\mathrm{J}$ integral theory. In: Kawata K, Akasaka T, editors. Proc Japan-US Conference, Composite Materials. Tokyo, 1981;171-80

[4] Zhang SY, Xiong DY. Crack propagation in the power-law nonlinear viscoelastic material. Appl Math Mech 1997;18(11):993-9

[5] Chandiramani JM, Librescu L, Aboudi J. The theory of orthotropic viscoelastic shear deformable composite flat panels and their dynamic stability. Int J Solids Struc 1989;25(5):465-82
[6] Zhang SY, Kitching R. Fracture toughness of unidirectional composite materials. Int Jour Mech Engng Edu 1990;18(1): 13-19

[7] Ashby MF et al. The fracture toughness of woods. Proc R Soc London A 1985;398:261-80

[8] Maiti SK. A relationship between the two in-plane fracture toughnesses of unidirectional fibre composites. Inter Jour Fracture 1986;32:R29-32

[9] Zhang SY. Damage, Failure, and Fracture of Resin-Based composites: Mechanisms, Theories, and Criteria. In: Cheremisinoff NP, editor. Handbook of Ceramics and Composites, vol. 2. New York:Marcel Dekker, 1992:51-104

[10] Whitney JM, Nuismer RJ. Stress fracture criteria for laminated composites containing stress concentrations. Jour Comp Mater $1974 ; 8: 253-65$ 